\title{
Elementary operations for quantum logic with a single trapped two-level cold ion beyond Lamb-Dicke limit
}

\author{
L. F. Wei, S. Y. Liu and X. L. Lei \\ Department of Physics, Shanghai Jiao Tong University, 1954 Huashan Road, Shanghai 200030, China
}

\begin{abstract}
A simple alternative scheme for implementing quantum gates with a single trapped cold twolevel ion beyond the Lamb-Dicke (LD) limit is proposed. Basing on the quantum dynamics for the laser-ion interaction described by a generalized Jaynes-Cummings model, one can introduce two kinds of elementary quantum operations i.e., the simple rotation on the bare atomic state, generated by applying a resonant pulse, and the joint operation on the internal and external degrees of the ion, performed by using an off-resonant pulse. Several typical quantum gates, including Hadamard gate, controlled-Z and controlled-NOT gates etc., can thus be implemented exactly by using these elementary operations. The experimental parameters including the LD parameter and the durations of the applied laser pulses, for these implementation are derived analytically and numerically. Neither the LD approximation for the laser-ion interaction nor the auxiliary atomic level is needed in the present scheme.
\end{abstract}

PACS number(s): 03.67.Lx; 42.50. Dv; 03.65.Bz

\section{INTRODUCTION}

Since Shor's algorithm for efficiently factoring large numbers was proposed [1], many studies have been carried out with a view to implement the quantum computation [2]. It has been shown that a simple rotation in the Hilbert space of an individual qubit, i.e. one-qubit gate, and a controlled rotation, such as a controlled-NOT $(C N)$ or controlled-Z $(C Z)$, between two different qubits, i.e. two-qubit controlled gate, are fundamental quantum gates. Any unitary transformation on arbitrarily many computational qubits can be carried out by repeatedly performing these fundamental gates [3]. Several kinds of physical systems, e.g. nuclear magnetic resonance (NMR) [1], coupled quantum dots [5] and the cavity QED [6] etc., have been proposed to implement these fundamental quantum gates. Quantum computation with trapped cold ions, introduced first by Cirac and Zoller [7], have been paid much attention theoretically and experimentally [17]. This scheme is based on the laser-ion interaction and possesses a long qubit coherence times [9]. Information is stored in the spin states of an array of trapped cold ions and manipulated by using laser pulses. In 1995, the fundamental quantum gates with a single trapped cold ion had been demonstrated experimentally [10]. In this scheme the ground and first excited states of the external vibration of the ion are used to encode the control qubit and the target qubit are encoded by two states of the internal degree freedom of the ion. However, the experimental operation involves an auxiliary atomic level and is limited in LD regime. Without performing the LD approximation and needing any auxiliary atomic level, some authors [11] 12] proposed another alternative method to realize another controlled operation, which is equivalent to the exact $C N$ logic operation 10] apart from phase factors, in a single trapped two-level cold ion by applying a single resonant pulse. In a recent work we proved that the exact $C N$ logic operation between the internal and external degrees of freedom of the ion can be realized assuredly by using a single off-resonant laser pulse, if the target qubit is encoded by the dressed atomic states [13. Adopting the usual encode scheme for conveniently measuring, i.e. the target qubit is encoded by the bare atomic states $(|g\rangle$ and $|e\rangle)$, we show in this paper that the fundamental quantum gates e.g. the Hadamard $(H)$ gate and the controlled-Z $(C Z)$ or $C N$ gate, can be implemented exactly in a single trapped ion register by using two kinds of elementary operations generated by applying different frequencies laser pulses. The experimental parameters for these realizations are derived by analytically and numerically. The multiquantum interaction between the ion and the applied laser is considered in the present work.

\section{ELEMENTARY OPERATIONS FOR MANIPULATING QUANTUM STATE OF LASER-ION SYSTEM BEYOND LAMB-DICKE LIMIT}

For simplicity, we assume that a single ion is stored in a coaxial resonator RF-ion trap [14], which provides pseudopotential oscillation frequencies satisfying the condition $\omega_{x}<<\omega_{y, z}$ along the principal axes of the trap. Only the quantized vibrational motion along $x$ direction is considered for the cooled ion [10]. Following Blockley et al [15], the interaction between a single trapped two-level cold ion and a classical single-mode travelling light field of frequency $\omega_{L}$ can be described by the following Hamiltonian: 


$$
\hat{H}(t)=\hbar \omega\left(\hat{a}^{\dagger} \hat{a}+\frac{1}{2}\right)+\frac{1}{2} \hbar \omega_{0} \hat{\sigma}_{z}+\frac{\hbar \Omega}{2}\left\{\hat{\sigma}_{+} \exp \left[i \eta\left(\hat{a}+\hat{a}^{\dagger}\right)-i\left(\omega_{L} t+\phi\right)\right]+\text { h.c. }\right\} .
$$

The first two terms correspond to the ion's kinetic and potential energy in the trap respectively, $\omega$ being the trap frequency. The final term gives the interaction between the ion and the light field with phase $\phi$. Pauli operators $\hat{\sigma}_{z}$ and $\hat{\sigma}_{ \pm}$describing the internal degrees of freedom of the ion. $\hat{a}^{\dagger}$ and $\hat{a}$ are the creation and annihilation operators of the trap vibrational quanta. $\omega_{0}$ is the atomic transition frequency, $\Omega$ is the Rabi frequency. $\eta(<1)$ is the LD parameter. Without loss of generality and for simplicity, we assumed that the laser is resonant with the $k$ th red-shifted vibrational sideband i.e. the frequency of laser field is chosen as $\omega_{L}=\omega_{0}-k \omega$. Under the usual rotating-wave approximation, the Hamiltonian of the system reads

$$
\hat{H}=\frac{\hbar \Omega}{2} \exp \left(-\frac{\eta^{2}}{2}-i \phi\right)\left\{\hat{\sigma}_{+}(i \eta)^{k}\left[\sum_{n=0}^{\infty} \frac{(i \eta)^{2 n} \hat{a}^{\dagger n} \hat{a}^{n}}{n !(n+k) !}\right] \hat{a}^{k}+h . c .\right\}
$$

in the interaction picture defined by the unitary operator $\hat{U}_{0}(t)=\exp \left[-i \omega t\left(\hat{a}^{\dagger} \hat{a}+1 / 2\right)\right] \exp \left(-i t \delta \hat{\sigma}_{z} / 2\right)$. Where $\delta=\omega_{0}-\omega_{L}$ is the detuning of laser field with the ion. The above Hamiltonian is similar to that of nonlinear coupled multiquantum Jaynes-Cummings model [16], which is exactly solvable. Therefore, it is easy to check the dynamical evolution of any two-qubit initial state by using evolution operator $\hat{U}(t)=\exp \left(-\frac{i}{\hbar} \hat{H} t\right)$. Indeed, with the help of relation [17] [18]

$$
\langle m-k|\langle e|\hat{H}| m\rangle| g\rangle=\left\{\begin{array}{l}
0, \quad m<k ; \\
\hbar i^{k} e^{-i \phi} \Omega_{m-k, m}, \quad m \geq k,
\end{array}\right.
$$

with

$$
\Omega_{m-k, m}=\frac{\Omega \eta^{k} e^{-\eta^{2} / 2}}{2} \sqrt{\frac{(m) !}{(m-k) !}} \sum_{n=0}^{m-k} \frac{(i \eta)^{2 n}}{(k+n) !} C_{m-k}^{n},
$$

the time evolution of the initial states $|m\rangle|e\rangle$ and $|m\rangle|g\rangle$ can be expressed as

$$
|m\rangle|e\rangle \longrightarrow \cos \Omega_{m, m+k} t|m\rangle|e\rangle-(-i)^{k-1} e^{i \phi} \sin \Omega_{m, m+k} t|m+k\rangle|g\rangle,
$$

and

$$
|m\rangle|g\rangle \longrightarrow\left\{\begin{array}{l}
|m\rangle|g\rangle, \quad m<k ; \\
\cos \Omega_{m-k, m} t|m\rangle|g\rangle+i^{k-1} e^{-i \phi} \sin \Omega_{m-k, m} t|m-k\rangle|e\rangle, \quad m \geq k,
\end{array}\right.
$$

respectively. The above treatment can also be modified directly to another laser excitation case, i.e., the $k$ th bluesideband laser addresses on the specifically chosen ion. In the present work only the red-sideband excitation is considered. It is seen from equations (3) and (4) that entangled states are caused from the time evolution of the state $|m\rangle|e\rangle$ and the state $|m\rangle|g\rangle$ with $m \geq k$. From this conditional quantum dynamics we can define two kinds of basic quantum operations: one is the simple rotations on the target qubit,

$$
\hat{r}_{m}(\phi, t)=\left\{\cos \Omega_{m, m} t|g\rangle\left\langle g\left|-i e^{-i \phi} \sin \Omega_{m, m} t\right| e\right\rangle\left\langle g\left|-i e^{i \phi} \sin \Omega_{m, m} t\right| g\right\rangle\left\langle e\left|+\cos \Omega_{m, m} t\right| e\right\rangle\langle e|\right\} \otimes|m\rangle\langle m|,
$$

generated by applying a resonant pulse $\left(\omega_{L}=\omega_{0}\right)$ to the ion, and another is the two-qubit joint operation

$$
\hat{R}_{m}(\phi, t)=\left\{\begin{array}{c}
|m\rangle|g\rangle\left\langlem \left|\left\langleg \left|+\left(\cos \Omega_{m, m+k} t|m\rangle|e\rangle-(-i)^{k-1} e^{i \phi} \sin \Omega_{m, m+k} t|m+k\rangle|g\rangle\right)\langle m|\langle e|, \quad m<k ;\right.\right.\right.\right. \\
\left(\cos \Omega_{m-k, m} t|m\rangle|g\rangle+i^{k-1} e^{-i \phi} \sin \Omega_{m-k, m} t|m-k\rangle|e\rangle\right)\langle m|\langle g| \\
\quad+\left(\cos \Omega_{m, m+k} t|m\rangle|e\rangle-(-i)^{k-1} e^{i \phi} \sin \Omega_{m, m+k} t|m+k\rangle|g\rangle\right)\langle m|\langle e|, \quad m \geq k,
\end{array}\right.
$$

performed by using an off-resonant pulse. Here $\phi$ and $t$ are the initial phase and duration of the applied pulse, respectively. We note that the state of the control qubit is unchanged during the operation $\hat{r}_{m}(\phi, t)$. It is worthwhile to point out that the qubit encoded by two Fock states of the external vibration of the ion can only be regarded as the control qubit, as all Fock states of the external vibration are present simultaneously. As a consequence, only the qubit encoded by two internal states of the ion can be serviced as the target qubit. Therefore, in what follows we only discuss how to implement the quantum operation on the internal state of the ion. 


\section{QUANTUM GATES WITH A SINGLE TRAPPED TWO-LEVEL COLD ION}

As we well know that computation on a register of qubits can be broken up into a series of two-qubit operations, accompanied by one-qubit rotations [3]. For the present system the operations $\hat{r}_{m}(\phi, t)$ and $\hat{R}_{m}(\phi, t)$ thus form a universal set i.e. any unitary operation on the internal state of the ion can be carried out by using the elements of the set repeatedly. Without the loss of generality, we now show how to implement three typical quantum logic operations : 1). Hadamard $(H)$ gate, 2). controlled-Z $(C Z)$ gate and 3). controlled-NOT $(C N)$ gate, respectively, in this simple quantum register.

Firstly, we discuss how to realize the $H$ gate operation

$$
\hat{H}_{m}=\frac{1}{\sqrt{2}}\{|g\rangle\langle g|+| e\rangle\langle g|+| g\rangle\langle e|-| e\rangle\langle e|\} \otimes|m\rangle\langle m| .
$$

It generates the uniform superposition of two encoded states of the target qubit from one of them. The control qubit remains in its initial state after this operation. The $H$ gate takes an important role in quantum computation, as almost all initial states of quantum computing are the uniform superposition of the encoded states the qubits. One can easily see from the discussion in Sec. II that the $H$ gate operation (7) can not be carried out exactly, if only the resonant laser pulse is applied to the ion. We now consider the combination operation $\hat{R}_{m}\left(\phi_{2}, t_{2}\right) \hat{r}_{m}\left(\phi_{1}, t_{1}\right)$ with $m<k$, It is performed by sequentially applying a resonant pulse and an off-resonant pulse. Under this operation the evolution of the initial states $|m\rangle|g\rangle$ and $|m\rangle|e\rangle$ can be expressed as

$$
\begin{aligned}
|m\rangle|g\rangle & \longrightarrow \hat{R}_{m}\left(\phi_{2}, t_{2}\right) \hat{r}_{m}\left(\phi_{1}, t_{1}\right)|m\rangle|g\rangle \\
& =\cos \Omega_{m, m} t_{1}|m\rangle|g\rangle-i e^{-i \phi_{1}} \sin \Omega_{m, m} t_{1}\left(\cos \Omega_{m, m+k} t_{2}|m\rangle|e\rangle-(-i)^{k-1} e^{i \phi_{2}} \sin \Omega_{m, m+k} t_{2}|m+k\rangle|g\rangle\right),
\end{aligned}
$$

and

$$
\begin{aligned}
|m\rangle|e\rangle & \longrightarrow \hat{R}_{m}\left(\phi_{2}, t_{2}\right) \hat{r}_{m}\left(\phi_{1}, t_{1}\right)|m\rangle|e\rangle \\
& =\cos \Omega_{m, m} t_{1}\left(\cos \Omega_{m, m+k} t_{2}|m\rangle|e\rangle-(-i)^{k-1} e^{i \phi_{2}} \sin \Omega_{m, m+k} t_{2}|m+k\rangle|g\rangle\right)-i e^{i \phi_{1}} \sin \Omega_{m, m} t_{1}|m\rangle|g\rangle,
\end{aligned}
$$

respectively. Obviously, if the following matching conditions

$$
\cos \Omega_{m, m} t_{1}=\frac{1}{\sqrt{2}}, \quad \cos \Omega_{m, m+k} t_{2}=-1, \quad \phi_{1}=\pi / 2 \pm 2 k \pi, k=1,2, \ldots,
$$

are satisfied, the $H$ gate $(7)$ is realized exactly. This mean that the exact $H$ gate operation can be implemented by sequentially applying a resonant pulse and an off-resonant pulse, i.e.,

$$
\hat{R}_{m}\left(T_{f}\right) \hat{r}_{m}\left(\phi_{r}, T_{r}\right) \longrightarrow \hat{H}_{m}
$$

with

$$
T_{f}=(\pi \pm 2 k \pi) / \Omega_{m, m+k}, \quad T_{r}=(\pi / 4 \pm 2 k \pi) / \Omega_{m, m}, \quad \phi_{r}=\pi / 2 \pm 2 k \pi .
$$

Similarly, one can prove also that

$$
\hat{r}_{m}\left(3 \pi / 2 \pm 2 k \pi, T_{r}\right) \hat{R}_{m}\left(T_{f}\right) \longrightarrow \hat{H}_{m}
$$

Therefore, the exact $H$ gate operation on the internal state of the ion can be realized by applying a resonant and an off-resonant pulses sequentially.

Secondly, we want to realize the typical two-qubit controlled gate e.g. the controlled-Z $(C Z)$ gate, which takes the form

$$
\hat{C} Z_{01}=|0\rangle|g\rangle\langle 0|\langle g|+| 0\rangle| e\rangle\langle 0|\langle e|+| 1\rangle| g\rangle\langle 1|\langle g|-| 1\rangle| e\rangle\langle 1|\langle e|=\left(\begin{array}{cccc}
1 & 0 & 0 & 0 \\
0 & 1 & 0 & 0 \\
0 & 0 & 1 & 0 \\
0 & 0 & 0 & -1
\end{array}\right)
$$


in the computational space $\Gamma: \Gamma=\{|0\rangle,|1\rangle\} \otimes\{|g\rangle,|e\rangle\}$. The control qubit in the present work is encoded by $|0\rangle$ and $|1\rangle$, i.e. the ground and first excited states of the external vibration of the ion. This gate is called also the controlledrotation $(C R)$ operation, which represents that the state of the target qubit is rotated by the Pauli operator $\sigma_{z}$ if and only if the control qubit is in the state $|1\rangle$. We now show that the exact $\hat{C Z} Z_{01}$ gate can be implemented exactly by a single off-resonant pulse, once the experimental parameters e.g. the LD parameter $\eta$ and duration of the applied pulse. In the computational space $\Gamma$ the two-qubit operation $\hat{R}_{m}(\phi, t)$, performed by using a $k$ th red-sideband pulse with initial phase $\phi$ and duration $t$, takes the following form

$$
\begin{aligned}
\hat{R}_{01}(\phi, t) & =|0\rangle|g\rangle\left\langle0 \left|\left\langleg \left|+\left(\cos \Omega_{0,1} t|0\rangle|e\rangle-e^{i \phi} \sin \Omega_{0,1} t|1\rangle|g\rangle\right)\langle 0|\langle e|\right.\right.\right.\right. \\
& +\left(\cos \Omega_{0,1}|1\rangle|g\rangle+e^{-i \phi} \sin \Omega_{0,1} t|0\rangle|e\rangle\right)\left\langle1 \left|\left\langleg \left|+\left(\cos \Omega_{1,2} t|1\rangle|e\rangle-e^{i \phi} \sin \Omega_{1,2} t|2\rangle|g\rangle\right)\langle 1|\langle e|\right.\right.\right.\right.
\end{aligned}
$$

for $k=1$, and

$$
\begin{aligned}
\hat{R}_{01}(\phi, t) & =|0\rangle|g\rangle\left\langle0 \left|\left\langleg \left|+\left(\cos \Omega_{0, k} t|0\rangle|e\rangle-(-i)^{k-1} e^{i \phi} \sin \Omega_{0, k} t|k\rangle|g\rangle\right)\langle 0|\langle e|\right.\right.\right.\right. \\
& +|1\rangle|g\rangle\left\langle1 \left|\left\langleg \left|+\left(\cos \Omega_{1, k+1} t|1\rangle|e\rangle-(-i)^{k-1} e^{i \phi} \sin \Omega_{1, k+1} t|k+1\rangle|g\rangle\right)\langle 1|\langle e|,\right.\right.\right.\right.
\end{aligned}
$$

for $k \geq 2$. Obviously, if the LD parameter $\eta$ and the duration of the applied off-resonant pulse are set up properly to satisfy the condition

$$
\cos \Omega_{0, k} t=1, \quad \cos \Omega_{1, k+1} t=-1,
$$

the two-qubit operation $\hat{R}_{01}$ reduces to the exact $C Z$ logic gate. It is worth noting that there is no any requirement on the initial phase of the applied pulse for realizing $C Z$ gate (13).

Finally, we turn to realize the exact controlled-NOT $(C N)$ logic gate with a single trapped two-level cold ion. This gate takes the form

$$
\hat{C N} N_{01}=|0\rangle|g\rangle\langle 0|\langle g|+| 0\rangle| e\rangle\langle 0|\langle e|+| 1\rangle| e\rangle\langle 1|\langle g|+| 1\rangle| g\rangle\langle 1|\langle e|=\left(\begin{array}{cccc}
1 & 0 & 0 & 0 \\
0 & 1 & 0 & 0 \\
0 & 0 & 0 & 1 \\
0 & 0 & 1 & 0
\end{array}\right)
$$

in the $\Gamma$ space defined above. It can also be called as controlled-X $(C X)$ gate, as it represents that the target qubit is rotated by Pauli operator $\sigma_{x}$ when the control qubit is in the state $|1\rangle$. Liking to the recent works [1] [12], we can easily show that a single resonant pulse may also result in a two-qubit controlled operation, i.e., a resonant pulse satisfying the condition

$$
\cos \Omega_{0,0} t=1, \quad \sin \Omega_{1,1} t=1,
$$

yields the controlled operation 11,12

$$
\hat{r}_{01}(\phi)=\left(\begin{array}{cccc}
1 & 0 & 0 & 0 \\
0 & 1 & 0 & 0 \\
0 & 0 & 0 & -i e^{i \phi} \\
0 & 0 & -i e^{-i \phi} & 0
\end{array}\right)
$$

which is equivalent to the exact $C N$ gate [10], apart from phase factors that must be eliminated by applying subsequent operations. In what follows we show how to implement the exact $C N$ gate with a single trapped two-level cold ion beyond LD limit by using three pulse sequentially. Indeed, one can easily prove that

$$
\hat{r}_{01}\left(\phi_{3}, t_{3}\right) \hat{C Z}{ }_{01} \hat{r}_{01}\left(\phi_{1}, t_{1}\right)=\left(\begin{array}{cccc}
A_{0000} & A_{0001} & 0 & 0 \\
A_{0100} & A_{0101} & 0 & 0 \\
0 & 0 & A_{1000} & A_{1011} \\
0 & 0 & A_{1110} & A_{1111}
\end{array}\right)
$$

with 


$$
\begin{aligned}
& A_{0000}=\cos \Omega_{0,0} t_{3} \cos \Omega_{0,0} t_{1}-e^{i\left(\phi_{3}-\phi_{1}\right)} \sin \Omega_{0,0} t_{3} \sin \Omega_{0,0} t_{1}, \\
& A_{0001}=-i e^{i \phi_{1}} \cos \Omega_{0,0} t_{3} \sin \Omega_{0,0} t_{1}-i e^{i \phi_{3}} \sin \Omega_{0,0} t_{3} \cos \Omega_{0,0} t_{1}, \\
& A_{0100}=-i e^{-i \phi_{3}} \sin \Omega_{0,0} t_{3} \cos \Omega_{0,0} t_{1}-i e^{-i \phi_{1}} \cos \Omega_{0,0} t_{3} \sin \Omega_{0,0} t_{1}, \\
& A_{0101}=\cos \Omega_{0,0} t_{3} \cos \Omega_{0,0} t_{1}-e^{-i\left(\phi_{3}-\phi_{1}\right)} \sin \Omega_{0,0} t_{3} \sin \Omega_{0,0} t_{1} \\
& A_{1010}=\cos \Omega_{1,1} t_{3} \cos \Omega_{1,1} t_{1}+e^{i\left(\phi_{3}-\phi_{1}\right)} \sin \Omega_{1,1} t_{3} \sin \Omega_{1,1} t_{1}, \\
& A_{1011}=-i e^{i \phi_{1}} \cos \Omega_{1,1} t_{3} \sin \Omega_{1,1} t_{1}+i e^{i \phi_{3}} \sin \Omega_{1,1} t_{3} \cos \Omega_{1,1} t_{1}, \\
& A_{1110}=-i e^{i \phi_{1}} \cos \Omega_{1,1} t_{3} \sin \Omega_{1,1} t_{1}+i e^{i \phi_{3}} \sin \Omega_{1,1} t_{3} \cos \Omega_{1,1} t_{1}, \\
& A_{1111}=-\cos \Omega_{1,1} t_{3} \cos \Omega_{1,1} t_{1}-e^{-i\left(\phi_{3}-\phi_{1}\right)} \sin \Omega_{1,1} t_{3} \sin \Omega_{1,1} t_{1},
\end{aligned}
$$

with $t_{1}$ and $t_{3}$ the durations of the first and third applied resonant laser pulses, respectively. If two initial phases $\phi_{1}, \phi_{3}$ satisfy the relation

$$
\phi_{3}-\phi_{1}= \pm 2 k \pi, \quad k=0,1,2, \ldots,
$$

the matrix elements on the right side of Eq. (19) become

$$
\left\{\begin{array}{l}
A_{0000}=A_{0101}=\cos \Omega_{0,0}\left(t_{3}+t_{1}\right), \quad A_{0001}=-i e^{i \phi_{1}} \sin \Omega_{0,0}\left(t_{3}+t_{1}\right), \quad A_{0100}=-i e^{-i \phi_{1}} \sin \Omega_{0,0}\left(t_{3}+t_{1}\right), \\
A_{0101}=-A_{1111}=\cos \Omega_{0,0}\left(t_{3}-t_{1}\right), \quad A_{1011}=i e^{i \phi_{1}} \sin \Omega_{1,1}\left(t_{3}-t_{1}\right), \quad A_{1110}=-i e^{-i \phi_{1}} \sin \Omega_{1,1}\left(t_{3}-t_{1}\right) .
\end{array}\right.
$$

Furthermore, if the following matching conditions

$$
\cos \Omega_{0,0}\left(t_{3}+t_{1}\right)=1, \quad \sin \Omega_{1,1}\left(t_{3}-t_{1}\right)=\left\{\begin{array}{l}
1 \quad \text { for } \phi_{1}=3 \pi / 2+2 k^{\prime} \pi, k^{\prime}=0,1,2, \ldots ; \\
-1 \text { for } \phi=\pi / 2+2 k^{\prime} \pi, k=0,1,2, \ldots
\end{array}\right.
$$

are satisfied, we have

$$
A_{0000}=A_{0101}=A_{1011}=A_{1110}=1, \quad A_{0001}=A_{0100}=A_{1000}=A_{1111}=0 .
$$

This means that, under the conditions (20) and (21), the operation (18) reduces to the exact reduced $C N$ logic gate [10], i.e.,

$$
\hat{r}_{01}\left(\phi_{3}, t_{3}\right) \hat{C Z} Z_{01} \hat{r}_{01}\left(\phi_{1}, t_{1}\right) \longrightarrow\left(\begin{array}{cccc}
1 & 0 & 0 & 0 \\
0 & 1 & 0 & 0 \\
0 & 0 & 0 & 1 \\
0 & 0 & 1 & 0
\end{array}\right)=\hat{C N} N_{01}
$$

Specifically, we now discuss how to set up the experimental parameters to realize the $\hat{C N}$ logic operation (17). These parameters include the LD parameter and the durations of the applied laser pulses. Firstly, the requisite LD parameter and the duration of the off-resonant red-sideband pulse for realizing the $C Z$ gate are determined by the equation (16), which implies

$$
\frac{\Omega_{1, k+1}}{\Omega_{0, k}}=\sqrt{k+1}-\frac{\eta^{2}}{\sqrt{k+1}}=\frac{q-0.5}{p}, \quad t=2 p \pi / \Omega_{0, k}=T_{z}, \quad p, q=1,2,3, \ldots,
$$

Secondly, using the LD parameter determined from the above equation, the durations of two resonant pulses surrounding the $k$ th red-sideband pulse should be determined by solving the equation (21) for their different initial phases, e.g.,

$$
\begin{cases}t_{1}=\pi\left(\frac{p^{\prime}+1}{\Omega_{0,0}}+\frac{q^{\prime}+0.25}{\Omega_{1,1}}\right), \quad t_{3}=\pi\left(\frac{p^{\prime}+1}{\Omega_{0,0}}-\frac{q^{\prime}+0.25}{\Omega_{1,1}}\right), & \text { for } \phi_{1}=\phi_{3}=\pi / 2 ; \\ t_{1}=\pi\left(\frac{p^{\prime}+1}{\Omega_{0,0}}-\frac{q^{\prime}+0.25}{\Omega_{1,1}}\right), \quad t_{3}=\pi\left(\frac{p^{\prime}+1}{\Omega_{0,0}}+\frac{q^{\prime}+0.25}{\Omega_{1,1}}\right), \text { for } \phi_{1}=\phi_{3}=3 \pi / 2 .\end{cases}
$$

Obviously, these parameters mightily are dependent of the frequency of the applied red-sideband laser light i.e., the choice of $k$, once the atomic transition frequency and trap frequency are defined. For different $k$ values, some values of these experimental parameters for this realization are given in Table I by numerical method. 
TABLE. some experimental parameters for realizing the exact CN gate by three-step sequential pulses with $\phi_{1}=\phi_{3}=\pi / 2$.

\begin{tabular}{|c|c|c|c|c|c|c|c|c|}
\hline & $\eta$ & $\Omega t_{2} / \pi$ & $\Omega t_{1} / \pi$ & $\Omega t_{3} / \pi$ & $\eta$ & $\Omega t_{2} / \pi$ & $\Omega t_{1} / \pi$ & $\Omega t_{3} / \pi$ \\
\hline & 0.96920 & 13.202 & 29.179 & 2.8108 & 0.56625 & 66.340 & 3.2117 & 1.4838 \\
\hline & & 39.607 & \begin{tabular}{|c|}
32.377 \\
\end{tabular} & 6.0098 & & & \begin{tabular}{|l}
9.0152 \\
\end{tabular} & 3.7584 \\
\hline & & 66.012 & \begin{tabular}{|l|}
35.576 \\
\end{tabular} & 9.2087 & & & \begin{tabular}{|l|}
13.711 \\
\end{tabular} & 5.0713 \\
\hline & & 118.82 & \begin{tabular}{|l|}
38.775 \\
\end{tabular} & 12.408 & & & \begin{tabular}{|l|}
19.514 \\
\end{tabular} & 3.9634 \\
\hline & & & \begin{tabular}{|l|}
41.974 \\
\end{tabular} & \begin{tabular}{|l|}
15.607 \\
\end{tabular} & & & \begin{tabular}{|l|}
24.211 \\
\end{tabular} & 8.6589 \\
\hline & & & 45.173 & \begin{tabular}{|l|}
18.805 \\
\end{tabular} & & & \begin{tabular}{|l|}
25.449 \\
\end{tabular} & 16.811 \\
\hline & & & & & & & & \\
\hline \multirow{6}{*}{\multicolumn{2}{|c|}{0.48191}} & 18.645 & 2.9777 & 1.5148 & 0.30135 & 128.90 & 2.6684 & 1.5174 \\
\hline & & 55.934 & \begin{tabular}{|l|}
5.2239 \\
\end{tabular} & \begin{tabular}{|l|}
3.7611 \\
\end{tabular} & & & \begin{tabular}{|l|}
6.8542 \\
\end{tabular} & 5.7032 \\
\hline & & $\ldots$ & 8.1496 & 8.3539 & & & 15.853 & 8.9029 \\
\hline & & & 13.322 & \begin{tabular}{|l|}
15.594 \\
\end{tabular} & & & \begin{tabular}{|l}
19.621 \\
\end{tabular} & 13.866 \\
\hline & & & 19.381 & 12.067 & & & 21.923 & 11.564 \\
\hline & & & $\cdots$ & \begin{tabular}{|l|}
$\cdots$ \\
\end{tabular} & & & $\cdots$ & $\cdots$ \\
\hline \multirow{6}{*}{\multicolumn{2}{|c|}{\begin{tabular}{|l|l|}
0.23549 \\
\end{tabular}}} & 69.853 & 2.6005 & \begin{tabular}{|l|}
1.5119 \\
\end{tabular} & 0.17379 & 327.14 & 2.5538 & 1.5070 \\
\hline & & $\ldots$ & \begin{tabular}{|l|}
4.6567 \\
\end{tabular} & 3.5678 & & & \begin{tabular}{|l|}
6.6147 \\
\end{tabular} & 5.5679 \\
\hline & & & 6.8337 & \begin{tabular}{|l|}
1.3913 \\
\end{tabular} & & & \begin{tabular}{|l|}
12.706 \\
\end{tabular} & 11.659 \\
\hline & & & 8.7692 & \begin{tabular}{|l|l|}
7.6807 \\
\end{tabular} & & & 16.831 & 11.596 \\
\hline & & & 12.882 & \begin{tabular}{|l|l|}
11.793 \\
\end{tabular} & & & 20.954 & 11.533 \\
\hline & & & $\begin{array}{ll}\cdots \\
\end{array}$ & \begin{tabular}{|l|}
$\cdots$ \\
\end{tabular} & & & \begin{tabular}{|l|}
$\cdots$ \\
\end{tabular} & $\cdots$ \\
\hline & $\cdots$ & $\cdots$ & $\cdots$ & $\cdots$ & $\cdots$ & $\cdots$ & $\cdots$ & $\cdots$ \\
\hline \multirow[t]{14}{*}{2} & \multirow[t]{7}{*}{0.7864} & 49.846 & 4.5099 & 0.9395 & 0.50753 & \begin{tabular}{|l|}
61.714 \\
\end{tabular} & 3.0410 & 1.5089 \\
\hline & & & \begin{tabular}{|l|}
9.9593 \\
\end{tabular} & 6.3889 & & & \begin{tabular}{|l}
7.5908 \\
\end{tabular} & 6.0587 \\
\hline & & & 18.133 & \begin{tabular}{|l|}
14.563 \\
\end{tabular} & & & \begin{tabular}{|l|}
12.141 \\
\end{tabular} & 10.608 \\
\hline & & & 23.583 & 20.012 & & & 14.416 & 12.883 \\
\hline & & & 33.448 & 15.596 & & & 16.690 & 15.158 \\
\hline & & & 43.314 & 11.180 & & & 22.030 & 14.369 \\
\hline & & & \begin{tabular}{|l|}
$\cdots$ \\
\end{tabular} & \begin{tabular}{|l|}
$\cdots$ \\
\end{tabular} & & & $\cdots$ & $\cdots$ \\
\hline & \multirow[t]{6}{*}{0.27778} & 609.54 & 2.6418 & 1.5156 & 0.81347 & 119.01 & 4.8421 & 0.72655 \\
\hline & & & \begin{tabular}{|l|}
6.9729 \\
\end{tabular} & \begin{tabular}{|l|}
1.3417 \\
\end{tabular} & & & \begin{tabular}{|l|}
10.411 \\
\end{tabular} & 6.2952 \\
\hline & & & 8.8778 & \begin{tabular}{|l|}
7.7516 \\
\end{tabular} & & & \begin{tabular}{|l}
13.195 \\
\end{tabular} & 9.0796 \\
\hline & & & 13.035 & \begin{tabular}{|l|}
11.909 \\
\end{tabular} & & & \begin{tabular}{|l|}
18.764 \\
\end{tabular} & 14.648 \\
\hline & & & 15.114 & 13.988 & & & \begin{tabular}{|l|}
21.548 \\
\end{tabular} & 17.433 \\
\hline & & & 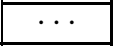 & $\cdots$ & & & $\cdots$ & $\cdots$ \\
\hline & & & $\cdots$ & $\cdots$ & & & $\cdots$ & $\cdots$ \\
\hline \multirow[t]{5}{*}{3} & \multirow[t]{4}{*}{0.70711} & 71.168 & 3.8521 & 1.2840 & 0.40825 & 939.48 & 2.8260 & 1.5217 \\
\hline & & 355.84 & 6.4201 & 3.8521 & & & 7.1736 & 5.8693 \\
\hline & & $\ldots$ & \begin{tabular}{|l|}
14.124 \\
\end{tabular} & \begin{tabular}{|l}
11.556 \\
\end{tabular} & & & \begin{tabular}{|l}
11.521 \\
\end{tabular} & $\begin{array}{l}10.217 \\
\end{array}$ \\
\hline & & & & & & & 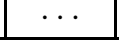 & \\
\hline & & & & & & & & \\
\hline
\end{tabular}

The durations of the applied pulses are given by the quantities $\Omega t_{j} / \pi, j=1,2,3$ in the table. It is seen from the table that the switching speed of the $C N$ gate depends on the Rabi frequency $\Omega$ and the LD parameter $\eta$. It may be estimated numerically For example, in the case of of a recent experiment [10] a single ${ }^{9} \mathrm{Be}^{+}$ion confined in a coaxialresonator radio frequency (RF)-ion trap and cooled to its quantum ground state by Ramman cooling, the target qubit is spanned by two ${ }^{2} S_{1 / 2}$ hyperfine ground states of ${ }^{9} \mathrm{Be}^{+}:{ }^{2} S_{1 / 2}\left|F=2, m_{F}=2\right\rangle$ and ${ }^{2} S_{1 / 2}\left|F=2, m_{F}=1\right\rangle$, separated by $\omega_{0} / 2 \pi=1.25 \mathrm{GHz}$. If the Rabi frequencies are chosen as [10] $\Omega=2 \pi \times 140 \mathrm{kHz}$ for resonant excitations and $\eta \Omega=2 \pi \times 30 \mathrm{kHz}$ for off-resonant excitations, one can easily show that the shortest duration of the applied pulses for the realization of the exact $C N$ gate is about $10^{-4} \mathrm{sec}$. (10 kHz order). We see also that the durations of the first and third (resonant) pulses are less than the second (off-resonant) one. So the switching speed of the $C N$ gate is mainly dependent of the speed of the two-qubit $C Z$ gate operation, which is approximately $10 \mathrm{kHz}$ order. This speed is the same as that of $C N$ gate demonstrated in [10]. 


\section{CONCLUSIONS AND DISCUSSIONS}

So far we have proposed a theoretical scheme to exactly implement several typical unitary operations on the internal state of a single trapped cold ion e.g. the Hadamard $(H)$, controlled-Z $(C Z)$ and controlled-NOT $(C N)$ or controlled-

$\mathrm{X}(C X)$ gates by using the basic one-qubit operation $\hat{r}_{m}(\phi, t)$ and two-qubit joint operation $\hat{R}_{m}(\phi, t)$. Once the coupling parameter $\eta$ is set up appropriately, we have shown that:

1). the $C Z$ logic operation can be implemented by using a single off-resonant pulse with duration $T_{z}$,

2 ). the $H$ gate operation can be realized by two-step sequential pulse: an off-resonant pulse and a resonant pulse with a defined initial phase, especialy,

3). an off-resoant pulse (for realizing the $C Z$ gate) surrounded by two resonant pulses (for simply rotating the state of the target) yields to the exact $C N$ logic operation.

Of course, the relevant durations of the applied pulses should be set up accurately also for these realizations.

Compared to other methods [10] 11] [12] for implementing the quantum logic operations with a single trapped ion, we emphasize that the present scheme has some advantages. First, although the same number of pulses are needed for realizing the $C N$ gate as that reported in [10], the auxiliary atomic level and LD approximation are not required in our scheme. Consequently, pluses with different polarizations are not needed and our scheme can operates beyond LD limit. Second, the controlled operation constructed in the present paper is the exact $C N$ gate, while the controlled operation implemented in [11] [12] is not the exact $C N$ operation, although it is equivalent to the exact $C N$ gate under local transformation. Subsequent pulses must be carried out in order to eliminate the additional phase factors [11]. So, our scheme provides an alternative method differing from the previous ones for realizing the exact quantum logic operations with a single trapped two-level cold ion. We hope it may be demonstrated in future experiment.

\section{ACKNOWLEDGMENTS}

This work was supported by the Young Science Foundation of Shanghai, China and the Natural Science Foundation of China.

[1] P. W. Shor, Proc. 35th Annual Symposium on Foundations of Computer Science (IEEE Press, Los Alamitos, CA, 1994)

[2] A. Ekert and R. Jozsa, Rev. Mod. Phys. 68(1995)733.

[3] A. Barenco et al, Phys. Rev. A 52(1995)3457.

[4] N. A. Gershenfeld and I. L. Chuang, Science 275 (1997)350; D. G. Cory, A. F. Fahmy, and T. F. Havel, Proc. Nat. Acad. Sci. 94 (1997)1634.

[5] D. Loss and D. P. Divincenzo, Phys. Rev. A 57 (1998)120; T. Tanamoto, Phys. Rev. A 61(2000)022305.

[6] Q. A. Truchette et al, Phys. Rev. Lett. 75(1995)4710.

[7] J. I. Cirac and P. Zoller, Phys. Rev. Lett. 74(1995)4091.

[8] S. B. Zheng, Opt. Comm., 180(2000)111; A. Steane, App. Phys. B 64 (1997)623; D. F. V. James, ibid 66(1998)181.

[9] D. Kielpinski, et al, Science 291 (2001)1013; F. Schmidt-Kaler, et al, J. Mod. Opt. 47(2000)2573.

[10] C. Monroe, D. M. Meekhof, B. E. King, W. M. Itano, and D. J. Wineland, Phys. Rev. Lett., $15(1995) 4714$.

[11] C. Monroe, D. Leibfried, B. E. King, D. M. Meekhof, W. M. Itano, and D. J. Wineland, Phys. Rev. A 55(1997)R2489.

[12] L. X. Li and G. C. Guo, J. Opt. B2(2000)62.

[13] L. F. Wei and X. L. Lei, J. Opt. B 2(2000)581.

[14] S. R. Jefferts, C. M. Monroe, E. W. Bell, and D. J. Wineland, Phys. Rev. A 51 (1995)3112.

[15] C. A. Blockley, D. F. Walls and H. Kisken, Europhys. Lett., 17 (1992)509.

[16] W. Vogel and R. L. de Matos Filho, Phys. Rev., A 52(1995)4214; W.Vogel and D. -G. Welsch, Phys. Rev. A 40 (1989)7113.

[17] A. Steane et al, Phys. Rev. A62 (2000)042305

[18] D. J. Wineland and Wayne M. Itano, Phys. Rev. A20 (1979)1521. 